(C) 2021, The Authors. Published by Elsevier Inc. and Fass Inc. on behalf of the American Dairy Science Association ${ }^{\circledR}$. This is an open access article under the CC BY-NC-ND license (http://creativecommons.org/licenses/by-nc-nd/4.0/).

\title{
Infrared milk analyzers: Milk urea nitrogen calibration
}

\author{
M. Portnoy, C. Coon, $(1)$ and D. M. Barbano* \\ Northeast Dairy Foods Research Center, Department of Food Science, Cornell University, Ithaca, NY 14853
}

\begin{abstract}
Our first objective was to redesign a modified 14-sample milk calibration sample set to obtain a welldistributed range of milk urea nitrogen (MUN) concentrations while maintaining orthogonality with variation in fat, protein, and lactose concentration. Our second objective was to determine the within- and betweenlaboratory variation in the enzymatic spectrophotometric method on the modified milk calibration samples and degree of uncertainty in MUN reference values, and then use the modified milk calibration samples to evaluate and improve the performance of mid-infrared partial least squares (PLS) models for prediction of MUN concentration in milk. Changes in the modified milk calibration sample formulation and manufacturing procedure were made to achieve the desired range of MUN concentrations. A spectrophotometric enzymatic reference method was used to determine MUN reference values, and the modified milk calibration samples were used to calibrate 3 mid-infrared milk analyzers. The within- and between-laboratory variation in the reference values for MUN were 0.43 and $0.77 \%$, respectively, and the average expanded analytical uncertainty for the mean MUN value of the 14-sample calibration set was (mean $\pm \mathrm{SD}$ ) $16.15 \mathrm{mg} / 100 \mathrm{~g} \pm 0.09$ of milk. After slope and intercept adjustment to achieve a mean difference of zero with the calibration samples, it could be seen that the standard deviation of the differences of predicted versus reference MUN values among 3 different instruments and their PLS models were quite different. The orthogonal sample set was used (1) to determine when a PLS model did not correctly model out the background variation in fat, true protein, or anhydrous lactose; (2) to calculate an intercorrection factor to eliminate that effect, and (3) to improve the model performance (i.e., $50 \%$ reduction in standard deviation of the difference between instrument predictions and reference chemistry values for MUN).
\end{abstract}

Received April 22, 2020.

Accepted January 25, 2021

*Corresponding author: dmb37@cornell.edu
Key words: infrared milk analyzer, calibration, modified milk, milk urea nitrogen

\section{INTRODUCTION}

Milk component concentrations are measured for payment testing, dairy herd improvement, and dairy product process control. Results from performancevalidated chemical methods, such as the Mojonnier ether extraction for milk fat (Barbano et al., 1988; AOAC International, 2019; method 989.05), Kjeldahl direct method for true protein nitrogen determination (Barbano et al., 1991; AOAC International, 2019; method 991.22), and the spectrophotometric enzymatic analysis for milk lactose (Lynch et al., 2007; AOAC International, 2019; method 2006.06), are commonly used to establish reference values for calibration of rapid testing methods such as mid-infrared (MIR) milk analyzers. The primary method for rapid testing of a large number of milk samples is MIR milk analyzers, which simultaneously obtain information of many milk components for a high quantity of milk samples at a rate of 50 to 600 samples per hour, depending on the model of the MIR milk analyzer.

Milk MIR instruments rely on the relationship between MIR light absorbance by milk component molecules and their concentrations in milk (Goulden, 1964). Fat, protein, and lactose can be determined using fixed filter wavelengths (Kaylegian et al., 2009), where a sample wavelength and a reference wavelength are defined for each of the 3 main milk solids components. Other minor components are most commonly determined by the use of partial least squares (PLS) prediction models (Haaland and Thomas, 1988).

For milk analysis using MIR, both the fixedwavelength approach and the PLS prediction model approach are secondary methods that require calibration (i.e., slope and intercept adjustment) with a set of calibration samples with known chemical reference values. The common approach for MIR calibration is to analyze a set of milk calibration samples with known reference values for each milk component with the MIR instrument to obtain predicted values. A new calibration slope and intercept for the predicted values is determined using least squares linear regression 
analysis of the known reference values as a function of the MIR intercorrected values for traditional filter models, or predicted values for PLS models. Linear regression slope and intercept adjustments are performed on each milk component to obtain slope- and interceptcorrected data (Kaylegian et al., 2006).

Milk MIR calibration samples can be made by selecting 10 or more preserved raw milks from different farms to try to obtain a good range of each component with sample-to-sample variation in component concentration for every milk component to be calibrated. In practice, this is difficult to achieve. An approach to production of calibration samples that achieves a wider range of concentrations, with evenly spaced levels within the range and orthogonal design, to uncouple natural correlations among milk components was described by Kaylegian et al. (2006). This is called the modified milk calibration sample approach and has been used successfully to provide wide, well-distributed, and orthogonal design calibration samples for fat, protein, and lactose calibration that has been used in many commercial and US Department of Agriculture (USDA) laboratories in the United States and is produced in the Cornell University pilot plant once every $4 \mathrm{wk}$.

Milk urea nitrogen is one of the minor milk components (i.e., in low concentration) that can be determined from infrared milk spectra using a PLS model (Lefier, 1996) and is used as proxy for BUN analysis. Data from MUN testing is produced by dairy herd improvementand payment testing-laboratories to gain a clearer understanding of the efficiency of nitrogen utilization by lactating dairy cows for protein feeding management (Roseler et al., 1993; Nousiainen et al., 2004). When MUN is too high, there can be negative effects on dairy cow reproductive efficiency (Ferguson and Chalupa, 1989; Larson et al., 1997; Rajala-Schultz et al., 2001). In addition, MUN testing and adjustment of protein content of dairy cattle rations to reduce MUN can be used to help lower dairy farm environmental impacts by reducing excretion of urea in urine of dairy cows (Kohn et al., 1997). Therefore, accurate MUN concentration information in a timely manner is of great importance for dairy herd feeding and reproduction management. The MIR milk analyzers provide a rapid cost-effective method to measure MUN. However, it is a challenge for a laboratory to try to obtain raw milks from 8 to 12 individual farms every 2 wk that have a wide range of fat, protein, lactose, and MUN to use as a milk calibration sample set for MIR milk analyzers.

Our first objective was to redesign the modified milk calibration sample set described by Kaylegian et al. (2006) to obtain a well-distributed range of MUN concentrations, while maintaining orthogonality with variation in fat, protein, and lactose concentration. Our second objective was to determine the within- and between-laboratory variation in the enzymatic spectrophotometric method on the modified milk calibration samples and degree of uncertainty in MUN reference values, and then use the modified milk calibration samples to evaluate and improve the performance of MIR PLS models for prediction of MUN.

\section{MATERIALS AND METHODS}

\section{Experimental Design}

The original modified milk calibration samples were used as described by Kaylegian et al. (2006), with some modifications designed to produce a calibration sample set with a wide range of fat, protein, lactose, and MUN with no correlation in change of concentration among these 4 parameters. The addition of the MUN parameter was done by selecting target MUN concentrations for each sample in the set in such a way that the MUN concentration from sample-to-sample varied independently from the other 3 parameters, and also achieved a wide range of MUN concentration (from about 8-20 $\mathrm{mg} / 100 \mathrm{~g}$ of milk) among the 14 milks with a relatively even distribution of MUN concentration levels across the range. This was achieved by starting with a bulk milk with a relatively low MUN concentration to use to make the sample set and then using combinations of the milk fractions (cream, UF retentate, UF permeate) plus some water, lactose monohydrate, and urea to achieve the target composition of each of the 14 samples in the set.

\section{Pilot Plant Production of Calibration Samples}

Production of Modified Milk Calibration Samples to Include MUN. The method of production for the modified milk calibration set described by Kaylegian et al. (2006) was used with the modifications described below. Most of the production procedure was similar to that of Kaylegian et al. (2006), where the main steps involved were as follows: milk pasteurization $\left(72^{\circ} \mathrm{C}\right.$ for $\left.15 \mathrm{~s}\right)$ of $1,275 \mathrm{~kg}$ of whole milk, overnight gravity separation of the pasteurized milk that produced gravity-separated cream (upper 10\%) and low-fat milk (lower 90\%) that was removed from cone bottom tanks, and centrifugal cream separation of the low-fat milk. The gravity cream phase was drained from the gravity separation tank in layers, where the first layer removed (bottom layer) contained about $10 \%$ of the gravity cream layer, and the remaining layers were about $20 \%$ each of the gravity cream layer. The 
Table 1. Default target compositions for each sample in the redesigned modified milk calibration set, including MUN

\begin{tabular}{lcccc}
\hline $\begin{array}{l}\text { Sample } \\
\text { number }\end{array}$ & $\begin{array}{c}\text { Fat } \\
(\mathrm{g} / 100 \mathrm{~g})\end{array}$ & $\begin{array}{c}\text { True protein } \\
(\mathrm{g} / 100 \mathrm{~g})\end{array}$ & $\begin{array}{c}\text { Anhydrous lactose } \\
(\mathrm{g} / 100 \mathrm{~g})\end{array}$ & $\begin{array}{c}\text { MUN } \\
(\mathrm{mg} / 100 \mathrm{~g})\end{array}$ \\
\hline 1 & 0.20 & 4.30 & 4.00 & 15.00 \\
2 & 0.62 & 2.27 & 4.55 & 16.00 \\
3 & 1.05 & 3.96 & 5.10 & 8.00 \\
4 & 1.47 & 2.61 & 4.96 & 11.00 \\
5 & 1.89 & 3.62 & 4.28 & 17.50 \\
6 & 2.32 & 2.95 & 4.55 & 8.50 \\
7 & 2.74 & 3.28 & 4.55 & 10.00 \\
8 & 3.16 & 3.12 & 4.68 & 10.00 \\
9 & 3.58 & 3.45 & 4.14 & 13.50 \\
10 & 4.01 & 2.78 & 4.82 & 18.70 \\
11 & 4.43 & 3.79 & 4.00 & 9.00 \\
12 & 4.85 & 2.44 & 5.55 & 12.00 \\
13 & 5.28 & 4.13 & & 14.00 \\
14 & 5.70 & 2.10 & & \\
\hline
\end{tabular}

bottom cream layer (first removed) was the lowest in fat, and the top layer was the highest. Each cream layer was tested for its fat content by MIR. Based on the MIR results for each cream layer, selected layers were combined to obtain a final cream ingredient with a fat content between 22 and $27 \%$. The top gravity cream layer was not used because milk somatic cells, spores, and bacteria were concentrated in this layer, as described by Caplan et al. (2013) and Geer and Barbano (2014). With combinations of these ingredients (gravity cream, UF permeate, and UF retentate), plus $\alpha$-lactose monohydrate and distilled water as needed to increase the lactose range, a set of 14 samples with a controlled concentration range of anhydrous lactose, true protein, and fat can be formulated. Given the composition of each ingredient and the target compositions of the 14 samples, an Excel (Microsoft Corp.) solver was used to create an orthogonal design, meaning that there was no correlation in the change in concentration of each component (fat, protein, and lactose) with each other within the 14 samples. Additionally, the concentrations of each component within the set was evenly distributed throughout its range to avoid high-leverage samples (Kaylegian et al., 2006). The modified milk calibration sample set described above had been made for approximately $20 \mathrm{yr}$ at the Cornell University pilot plant facilities once every $4 \mathrm{wk}$ for the purpose of the USDA milk proficiency testing for chemical methods (Wojciechowski et al., 2016) and as a set for calibrating MIR milk analyzers.

Redesign of the Calibration Set. The concentrations of fat, protein, and anhydrous lactose for each of the 14 samples in the modified milk calibration set described by Kaylegian et al. (2006) was unchanged. The addition of the MUN parameter was done by selecting target MUN concentrations for each sample in the set in such a way that the MUN concentration from sample-to-sample varied independently from the other 3 parameters. A new Excel solver spreadsheet was created to calculate the amount of each ingredient required for each of the 14 samples within the set to achieve the target composition for each sample. Therefore, just as fat, protein, and lactose variations from sample-to-sample were uncorrelated, so was the variation in MUN to these 3 components. The default target composition of each sample for the redesigned sample set is shown in Table 1.

Challenges Faced When Including MUN in the Design. The production of modified milk calibration samples (Kaylegian et al., 2006), with a modification to include MUN as a fourth component, required an approach for reaching lower and higher MUN concentrations in certain samples to obtain a wide concentration range in the set. Additionally, the normal variation from month to month in MUN levels of the raw milk used to formulate the samples had to be considered. Variation in feeding and feeding management can affect the level of MUN in the starting raw milk (bulk tank milk from Cornell University Dairy) used in the sample production process. Therefore, modifications to the procedure had to ensure the ability to obtain a wide, well-distributed, orthogonal design of all 4 components within the set every month, regardless of the changes in the starting milk MUN levels. The main steps for production were (1) milk pasteurization $\left(72^{\circ} \mathrm{C}\right.$ for $15 \mathrm{~s}$ ) of 1,275 kg of whole milk; (2) overnight gravity separation of the pasteurized whole milk that produced gravity-separated cream (upper 10\%) and low-fat milk (lower 90\%) that was removed from the bottom of the cone-shaped tank; (3) centrifugal cream separation of the low-fat milk to produce skim milk; and (4) UF of the skim to produce a UF retentate with 2.5 times the 
protein concentration of the skim milk and a proteinfree UF permeate.

Obtaining a Wide Range of MUN Concentrations. To reach higher MUN concentrations, pure urea (Urea, U15-500; Thermo Fisher Scientific)was added to selected samples based on the calculation by the Excel solver to achieve MUN levels higher than the starting bulk milk. To reach lower MUN concentrations in the set, an ingredient with a lower MUN concentration was needed. The simplest ingredients to reduce MUN concentration were UF retentate and water. Adding too much water as an ingredient causes difficulties in maintaining good calibrations for the various milk solids parameters (i.e., total solids, solids not fat, and other solids). To minimize the need to add water to achieve lower MUN concentrations in some samples, a modification of the skim milk UF procedure was made to increase the target protein concentration in the UF retentate from about $6 \%$ (used by Kaylegian et al., 2006), which achieved a retentate protein content 2 times greater than the protein content of the starting milk, to approximately $8.2 \%$ true protein to produce a retentate with a lower MUN content (the selection of this protein value is explained in the following section). The removal of more UF permeate from the skim milk resulted in an increased ratio of protein to MUN in the UF retentate ingredient used to formulate the calibration samples. The MUN concentration of the permeate remained unchanged. This change in the UF process helped achieve lower MUN concentration in some samples without excessive addition of water.

Finding the Optimum Protein Concentration for the UF Retentate Ingredient. A sensitivity analysis was performed by calculating different retentate protein concentrations using different starting whole milk MUN concentrations in the Excel solver to find an optimum retentate protein concentration. This retentate protein concentration had to be high enough (had enough permeate removed) to be able to reach the low end of the MUN range in the sample set and high enough for the formulations to be less vulnerable to the month-to-month raw milk MUN variations. However, the protein concentration in the UF retentate could not be too high in order to avoid large additions of water and $\alpha$-lactose monohydrate in the formulations. This optimum protein concentration of the UF skim retentate was $8.2 \%$.

Nonetheless, during the formulation of the calibration samples, even with the carefully selected retentate protein level, there was still the issue of variation in the MUN content of the starting raw milk used to make calibration samples. The goal should be to source a bulk raw milk that has a MUN content between 8 and $12 \mathrm{mg}$ of MUN/100 of g milk to use for calibration sample production. In our study, the Excel solver was designed using the assumption that the bulk milk contained $10.5 \mathrm{mg}$ of MUN/100 g of milk. By always using a default value of $10.5 \mathrm{mg}$ of MUN/100 $\mathrm{g}$ of milk, regardless of what the actual MUN content is of the bulk raw milk, this maintains the absolute value of the range of MUN concentrations (i.e., absolute difference in $\mathrm{mg} / 100 \mathrm{~g}$ of milk) relatively constant with equal intervals of MUN concentrations within the range. As a result, the mean MUN concentration of the set will shift up and down slightly from batch-to-batch of calibration samples. When the MUN of the raw milk differed from $10.5 \mathrm{mg} / 100 \mathrm{~g}$ of milk by a large amount, then the proportion of water in the formulation of the 14 samples would not be affected, but the mean MUN concentration for the set would be shifted higher or lower, with the width of the range of concentrations remaining about the same. For example, in the case of bulk tank milk with MUN concentration of $9.38 \mathrm{mg} / 100$ $\mathrm{g}$ of milk, the MUN range of the set would go from 9.71 to $21.75 \mathrm{mg}$ of MUN/100 g of milk (absolute difference $=12.04$, mean $=14.77)$; however, if the bulk tank milk MUN concentration was $11.16 \mathrm{mg}$ of MUN/100 g of milk, the MUN range of the set would go from 11.38 to $23.51 \mathrm{mg}$ of MUN/100 $\mathrm{g}$ of milk (absolute difference = 12.13 , mean $=16.45$ ).

Calculation of Formulations. An Excel linear solver function was used to determine the formulations for the samples, similar to the solver described by Kaylegian, et al. (2006). However, the new solver was designed to include 4 parameters for the 14 samples, instead of 3. The solver input data (Table 2) was the composition of each ingredient (gravity cream, UF retentate, UF permeate, lactose monohydrate, and urea), and the minimum and maximum values of fat, true protein, anhydrous lactose, and MUN that were desired in the sample set. Percentiles within the specified concentration range of each component were chosen to result in the target concentrations for each sample (shown in Table 1), which were selected to achieve an orthogonal set. The output of the solver indicates the weight of each ingredient required for each of the 14 samples for a specific batch size (Table 3).

\section{Reference Chemical Analysis for Primary Milk Components}

Reference values for the primary milk components of the calibration samples were determined using the following validated methods: Mojonnier ether extraction for fat (Barbano et al., 1988; AOAC International, 2019; method 989.05); Kjeldahl direct method 
Table 2. Example of Excel Solver (Microsoft Corp.) input from April 29, 2019, where the composition of each ingredient was entered

\begin{tabular}{lcccc}
\hline Ingredient & $\begin{array}{c}\text { Fat } \\
(\mathrm{g} / 100 \mathrm{~g})\end{array}$ & $\begin{array}{c}\text { Protein } \\
(\mathrm{g} / 100 \mathrm{~g})\end{array}$ & $\begin{array}{c}\text { Anhydrous lactose } \\
(\mathrm{g} / 100 \mathrm{~g})\end{array}$ & MUN (mg/100 g) \\
\hline Cream & 23.4000 & 2.4400 & 3.6400 & 8.6297 \\
Retentate & 0.1500 & 7.9451 & 4.5690 & 10.0057 \\
Permeate & 0.0000 & 0.0700 & 4.7585 & 6.0034 \\
Lactose monohydrate & 0.0000 & 0.0000 & 95.0000 & 0.0000 \\
Urea & 0.0000 & 0.0000 & 0.0000 & $46,640.0000$ \\
Water & 0.0000 & 0.0000 & 0.0000 & 0.0000 \\
\hline
\end{tabular}

for true protein nitrogen determination (Barbano et al., 1991; AOAC International, 2019; method 991.22); spectrophotometric enzymatic analysis for lactose (Lynch et al., 2007; AOAC International, 2019; method 2006.06).

\section{Reference Chemical Analysis for MUN}

Reference values for MUN were determined using the enzymatic spectrophotometric urea determination method described below.

Principle. Milk was first diluted with a reagent (trichloroacetic acid) designed to precipitate fat and protein, which are removed by filtration. The method used for quantifying MUN is based on 2 main reactions. In the first reaction, urea and water form ammonia and carbon dioxide in the presence of the enzyme urease (EC 3.5.1.5). In the second reaction, ammonia (as ammonium ions) reacts with 2-oxoglutarate in the presence of reduced NADPH and the enzyme glutamate dehydrogenase (EC 1.4.1.2) to form L-glutamic acid, water, and $\mathrm{NADP}^{+}$. The molecule that absorbs light during the spectrophotometric measurement at
$340 \mathrm{~nm}$ is NADPH. Using Beer's Law, with the difference of absorption before and after the reactions have taken place, the amount of NADPH that was consumed can be determined. Therefore, by knowing how much NADPH was consumed, the amount of urea that was present can be calculated stoichiometrically. From the amount of urea, the amount of MUN can be calculated.

Reagents and Apparatus. A $0.3 \mathrm{M}$ trichloroacetic acid (Sigma-Aldrich, T6399-500G) solution was made. Reagents for the enzymatic assay procedure came from the Megazyme Urea/Ammonia Assay Kit (Rapid; product code K-URAMR). The kit included the following reagents: buffer $(18 \mathrm{~mL}, \mathrm{pH} 8.0)$ plus 2 -oxoglutarate and sodium azide $(0.02 \% \mathrm{wt} / \mathrm{vol})$ as a preservative, NADPH solution (which was dissolved in $12 \mathrm{~mL}$ of distilled water and portioned out to $1-\mathrm{mL}$ aliquots into flex tubes; VWR, 21008-959), glutamate dehydrogenase suspension, urease solution, and urea control powder to make a urea standard solution for the enzymatic analysis. A UV/vis Spectrophotometer (UV-1800 120V, Shimadzu Corporation) was used for the measurement of NADPH absorbance at $340 \mathrm{~nm}$.

Table 3. Example of the Excel Solver (Microsoft Corp.) output from April 29, 2019; the amount of each ingredient is shown for the formulation of each 14,960-g batch of each of the 14 samples in the set

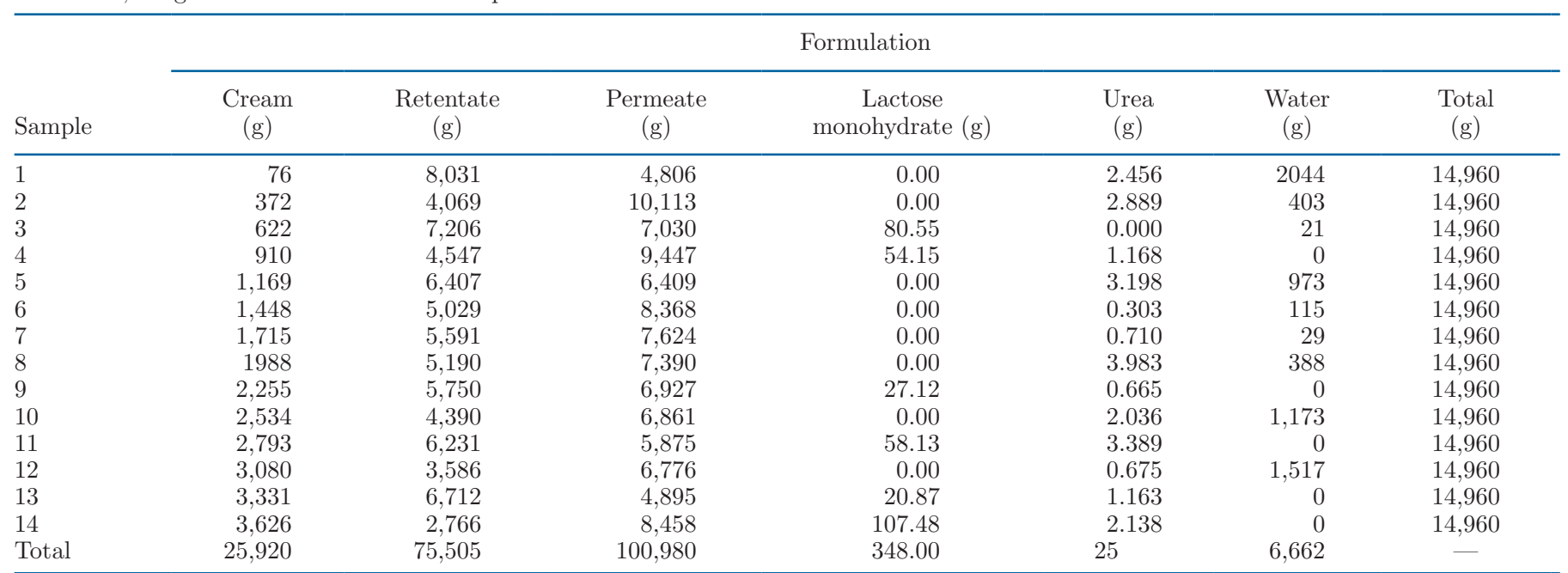


Statistical Analysis for Reference Chemistry Data. For performance statistical analysis of the MUN enzymatic spectrophotometric method, the AOAC International statistical procedures were used to determine within-laboratory statistics, referring to the method repeatability $\left[\mathrm{s}_{\mathrm{r}}=\right.$ within-laboratory repeatability standard deviation; RSDr = within-laboratory relative repeatability standard deviation $=\left(\mathrm{s}_{\mathrm{r}} /\right.$ mean $)$; r-value $=$ within-laboratory repeatability value $(2.8 \times$ $\left.\mathrm{s}_{\mathrm{r}}\right)$ ], as well as between-laboratory statistics, referring to the method reproducibility $\left[\mathrm{s}_{\mathrm{R}}=\right.$ between-laboratory reproducibility standard deviation; $\mathrm{RSD}_{\mathrm{R}}=$ betweenlaboratory relative reproducibility standard deviation $=\left(\mathrm{s}_{\mathrm{R}} /\right.$ mean $) ; \mathrm{R}$-value $=$ between-laboratory reproducibility value $\left.\left(2.8 \times \mathrm{s}_{\mathrm{R}}\right)\right]$. The meaning and relevance of each of these values is explained by Lynch (1998). The Microsoft Excel AOAC International Interlaboratory Statistical Program, version 1.11, was used to calculate these statistics and identify outliers following the guidelines for Interlaboratory Studies of AOAC International (AOAC International, 2019), as performed by Wojciechowski et al. (2016). Statistical outliers were identified using the Cochran, single Grubbs, and double Grubbs tests with a significance level of $2.5 \%$. Standard uncertainty and expanded uncertainty of chemical reference values were calculated as described by Ellison et al. (2000).

\section{Mid-Infrared Analysis of the Redesigned Modified Milk Calibration Samples}

The concentrations of the primary milk components (lactose, fat, and protein) and MUN in the redesigned modified milk calibration sets were also determined using milk MIR instruments for the purpose of creating a calibration curve to determine and perform secondary slope and intercept adjustment. The primary components were determined using the virtual filters of fat A, fat $B$, protein, and lactose with their respective sample and reference wavelengths (Kaylegian et al., 2009). The MUN was determined by PLS models, which varied by instrument model and manufacturer.

\section{Infrared Analysis}

Instrument Precalibration. The 3 infrared milk analyzers used to determine MUN in this study were precalibrated, as explained by Lynch et al. (2006), to ensure they were functioning appropriately, electrically and mechanically, before calibration. The wavelengths used for fat A, fat B, lactose, and protein were the optimized center wavelengths and band width reported by Kaylegian et al. (2009).
Calibration. Nine laboratories ran reference chemistry for fat, true protein, lactose, and MUN, using the reference methods mentioned previously, and an all-laboratory mean was calculated for each component in each sample in the modified milk calibration sample set (Kaylegian et al., 2006). Calibration (adjustment of secondary slope and intercept) for each instrument was performed for fat A, fat B, protein, lactose, and MUN, using the all-laboratory mean reference chemistry values for the redesigned modified milk calibration samples.

Evaluation of MUN PLS Model Performance. The redesigned modified milk calibration sets, which included the MUN component, were analyzed on 3 MIR instruments in 3 different laboratories, and data for fat, true protein, lactose, and MUN were reported. The instruments were from different instrument manufacturers and used different MUN PLS models.

\section{RESULTS AND DISCUSSION}

\section{Calibration Set Formulation and Reference Chemistry}

The redesigned modified milk calibration set was formulated using an Excel linear solver spreadsheet, where the concentrations of each component in each ingredient (combined cream, UF retentate, UF permeate, lactose monohydrate, urea, and water) were the inputs. These inputs are shown in Table 2, where the ingredient concentrations for the sample set formulated on April 29, 2019 are used as an example. The cream contained $23.40 \mathrm{~g}$ of fat/100 g, $2.44 \mathrm{~g}$ of protein/100 $\mathrm{g}$, and $3.64 \mathrm{~g}$ of lactose/100 g; the retentate contained $0.15 \mathrm{~g}$ of fat $/ 100 \mathrm{~g}$ (this value was kept constant from month to month), $7.95 \mathrm{~g}$ of protein $/ 100 \mathrm{~g}$, and $4.57 \mathrm{~g}$ of lactose/100 g; the permeate contained zero fat (this value was kept constant from month to month), 0.07 $\mathrm{g}$ of protein/100 $\mathrm{g}$ (this value was kept constant from month to month), and $4.76 \mathrm{~g}$ of lactose/100 g. The pure lactose monohydrate ingredient contained $95 \mathrm{~g}$ of anhydrous lactose $/ 100 \mathrm{~g}$, and the pure urea ingredient contained $46.64 \mathrm{~g}$ nitrogen/100 g (these values were kept constant from month to month). The MUN values for the cream, retentate, and permeate were kept constant from month to month in an effort to avoid issues in being able to reach the desired target concentrations on months when the starting milk MUN was too high or too low (as explained previously). The values that were adjusted every month were obtained from MIR instrument predictions when the ingredients were run on the MIR right after they were made in the pilot plant to obtain the concentration values and input them into the solver. 
Table 4. Reference chemistry values for the redesigned 14-sample modified milk calibration set made Apr. 29, 2019, including MUN, and method within-laboratory repeatability and between-laboratory reproducibility performance statistics for each chemical method

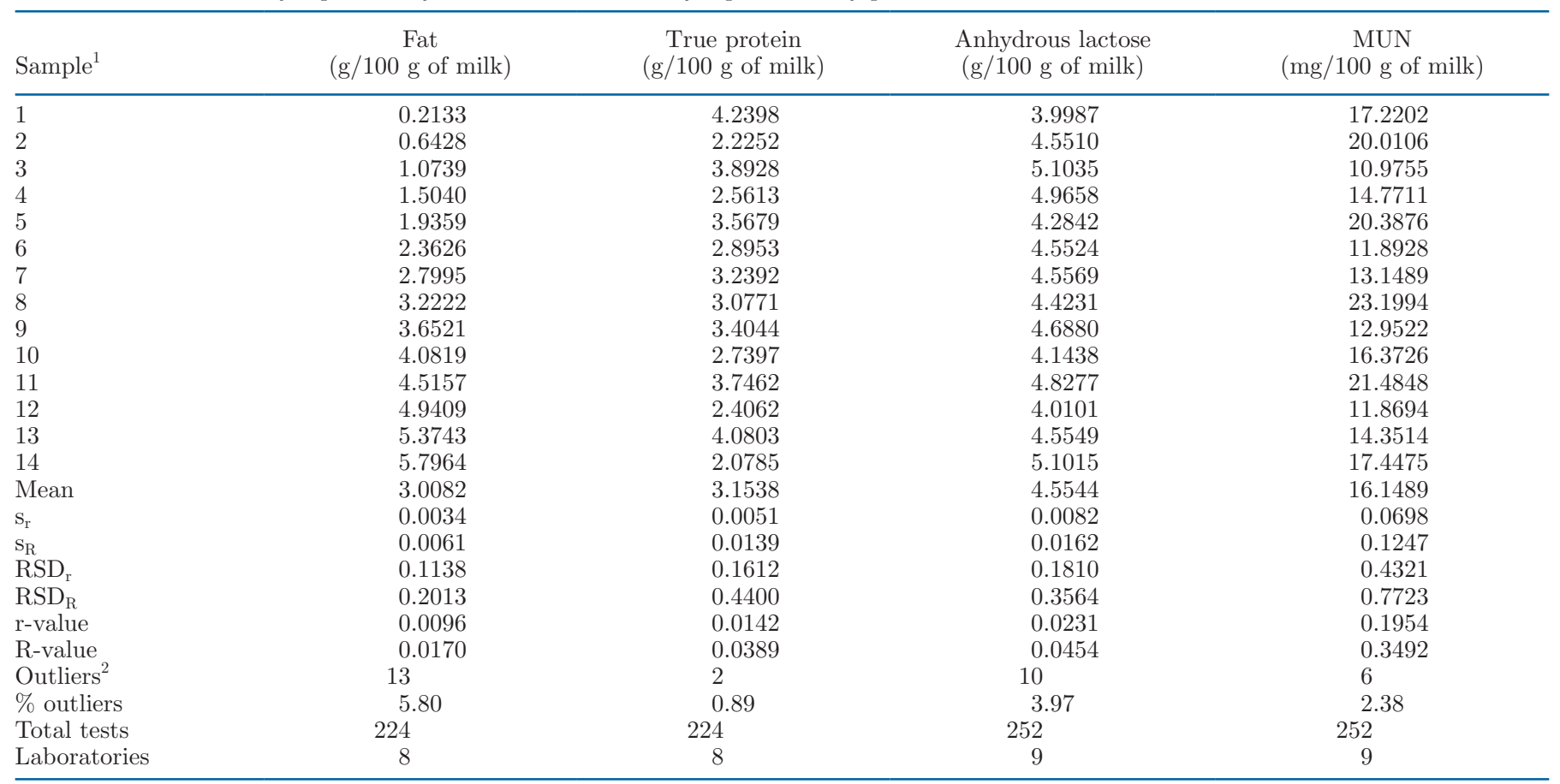

${ }^{1}$ Mean component concentrations, SD of repeatability $\left(\mathrm{S}_{\mathrm{r}}\right)$, SD of reproducibility $\left(\mathrm{s}_{\mathrm{R}}\right)$, repeatability value ( $\mathrm{r}$-value), and reproducibility value (R-value) are expressed in terms of $\mathrm{g} / 100 \mathrm{~g}$ ( or $\mathrm{mg} / 100 \mathrm{~g}$ for MUN) of milk. Relative SD of repeatability (RSD ${ }_{\mathrm{r}}$ ) and relative SD of reproducibility $\left(\mathrm{RSD}_{\mathrm{R}}\right)$ are expressed as percentages.

${ }^{2}$ Outlier removal at $P<0.025$.

The target concentrations and amount to be made of each sample were also specified in the spreadsheet before running the solver. Once the solver was run, the output included the amount of each ingredient needed to formulate each sample for the batch size that was specified. An example of this output is shown in Table 3 , where the output values from the sample set made on April 29, 2019, are used as an example. The weight of each ingredient that was needed to formulate each sample is shown in Table 3.

Once the sample set was made, split into sample vials (about 312 sets of 14 different milks each), and sent to the collaborating laboratories, each laboratory ran reference chemistry on the set for each component using the AOAC International reference methods for fat, true protein, and lactose, as described by Kaylegian et al. (2006), and the enzymatic spectrophotometric method for MUN, as described above. An all-laboratory mean was calculated (Wojciechowski et al., 2016) for each component of each sample, and these were the reference values used to calibrate the MIR instruments. These all-laboratory mean reference values are shown in Table 4 for the sample set made on April 29, 2019. Table 4 also contains the method performance statis- tics for each of the chemical methods, calculated as described by Wojciechowski et al. (2016). Both relative repeatability within a laboratory $\left(\mathrm{RSD}_{\mathrm{r}}\right)$ and relative reproducibility among laboratories $\left(\mathrm{RSD}_{\mathrm{R}}\right)$ were $<1 \%$ of the mean reference chemical value, indicating that all 4 methods performed well. The expanded uncertainty values for each reference chemical method of each sample are shown in Table 5. The mean expanded uncertainty values for fat, true protein, and anhydrous lactose are typical of values from month to month. The expanded uncertainty of MUN reference values for the 14 individual milks in the calibration set ranged from 0.058 to $0.117 \mathrm{mg}$ of MUN/100 g of milk. These values indicate the $95 \%$ confidence interval $( \pm)$ around the mean reference value.

\section{Use of the Modified Milk Calibration Set for MUN PLS Model Diagnostics}

The milk calibration sample set with the modification to include a wide, well-distributed range of MUN that is orthogonal with respect to fat, protein, and lactose can also be used to identify weaknesses in MUN PLS prediction models used on MIR milk analyzers. Because 
the 14 modified milk calibration set of samples have a wide range of MUN concentrations, but still maintain orthogonality in the set relative to the concentrations of the other main milk components (fat, protein, and lactose), correlations between the residual differences of MUN predictions by a PLS model minus reference chemistry MUN from each instrument as a function of variation in the concentration of each primary milk component can be used to determine if the PLS model for MUN is modeling out the effect of background sample matrix variation in fat, protein, and lactose in the matrix. For example, a graph of the residual differences between MUN instrument prediction minus all-laboratory mean MUN reference chemistry for each of the 14 samples plotted as a function of reference chemistry fat concentration is shown in Figure 1. As fat concentration in the samples increases the residual difference between instrument-predicted MUN and MUN, reference chemistry increases $\left(P<0.05 ; \mathrm{R}^{2}=\right.$ $0.65)$ and indicates that the MUN PLS model from MIR instrument 1 does not correctly "model-out" the effect of background variation in fat concentration. If the MUN PLS model for instrument 1 was correctly compensating for background variation in fat content, the slope of the residual plot would not be significantly different from zero. Another example is shown in Figure 2 for a different PLS model from MIR instrument 2. The failure of the MUN PLS model to model out the effect of background variation was a similar weakness as observed in instrument 1 (Figure 1), except that the PLS model in instrument 2 was not correctly modeling out the background variation in true protein, as for fat in instrument 1 . Instrument 1 and 2 are from 2 different instrument manufacturers and use different MUN PLS models.
The PLS model diagnostic graphs for a different PLS model from MIR instrument 3 are shown in Figures 3,4 , and 5. Lower correlations among the differences in MUN values (PLS MUN value minus reference MUN value) and the concentrations of each primary milk component (i.e., true protein, fat, and anhydrous lactose) are shown in Figures 3, 4 and 5, respectively. The slopes of the regression lines were not significantly different from zero $(P>0.05)$, indicating that the PLS model used on instrument 3 was working well.

\section{Correcting for Weaknesses in PLS Models}

In general, if PLS models developed by an equipment manufacturer and run on their commercial infrared milk analyzers have weaknesses, then the weaknesses in the PLS model needs to be corrected by the instrument manufacturer. This would be done by adding new spectra and the matching reference chemistry to the set of spectra and reference chemistry that was used to make the original PLS model. In the case of the example in Figure 2 (instrument 2) where the effect of background variation of protein is not modeled out correctly, the instrument manufacturer would need to obtain additional samples to add to the modeling set to correct the weakness. The fundamental problem is that in the population of modeling samples, if the PLS modeler performs a linear regression of the MUN as a function of protein concentration, the result would be a fairly strong correlation of increasing MUN as true protein increases in the samples in the PLS modeling set. Samples that break that correlation need to be added to the PLS modeling set. This will help reduce this weakness to background variation in protein in the new PLS model.

Table 5. Expanded (95\% CI) uncertainty $( \pm)$ around mean reference value for each sample for each component calculated, as described by Ellison et al. (2000)

\begin{tabular}{lcccc}
\hline Sample & $\begin{array}{c}\text { Fat } \\
(\mathrm{g} / 100 \mathrm{~g} \text { of milk })\end{array}$ & $\begin{array}{c}\text { Protein } \\
(\mathrm{g} / 100 \mathrm{~g} \text { of milk })\end{array}$ & $\begin{array}{c}\text { Lactose } \\
(\mathrm{g} / 100 \mathrm{~g} \text { of milk })\end{array}$ & $\begin{array}{c}\text { MUN } \\
(\mathrm{mg} / 100 \mathrm{~g} \text { of milk })\end{array}$ \\
\hline 1 & 0.0040 & 0.0196 & 0.0093 & 0.0737 \\
2 & 0.0031 & 0.0076 & 0.0132 & 0.0577 \\
3 & 0.0031 & 0.0121 & 0.0151 & 0.1010 \\
4 & 0.0026 & 0.0084 & 0.0142 & 0.0805 \\
5 & 0.0019 & 0.0133 & 0.0119 & 0.0904 \\
6 & 0.0028 & 0.0088 & 0.0116 & 0.0922 \\
7 & 0.0015 & 0.0062 & 0.0082 & 0.1166 \\
8 & 0.0030 & 0.0108 & 0.0062 & 0.0953 \\
9 & 0.0044 & 0.0116 & 0.0142 & 0.0576 \\
10 & 0.0034 & 0.0102 & 0.0081 & 0.0775 \\
11 & 0.0066 & 0.0119 & 0.0056 & 0.0923 \\
12 & 0.0074 & 0.0100 & 0.0114 & 0.1117 \\
13 & 0.0094 & 0.0134 & 0.0148 & 0.0883 \\
14 & 0.0050 & 0.0078 & 0.0154 & 0.0118 \\
\hline
\end{tabular}


Fat on MUN

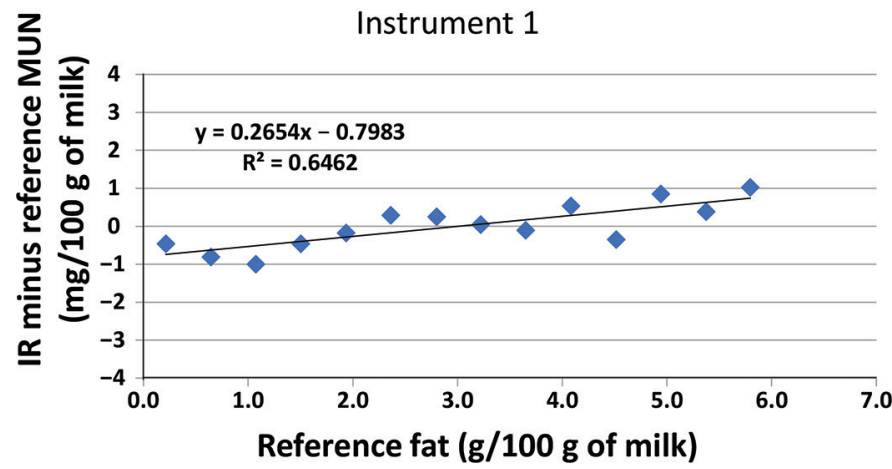

Figure 1. Partial least squares (PLS) model diagnostic graph for instrument 1, showing the difference between the MUN PLS model prediction value and the MUN reference value versus the reference chemistry concentration values for fat of the modified milk calibration samples from April 29, 2019. IR = infrared.

Is there anything that a user can do when they have a manufacturer-provided PLS model that has the type of weakness similar to instrument 2 that is shown in Figure 2? Using the 14-sample modified milk calibration set described in the current study, a calculated correction to a PLS model could be created by a user as a calculated adjustment because the effect is so consistent and large. In fact, even though developing a better PLS model by the instrument manufacturer would be the best solution, by knowing which primary component is not being "modeled-out" correctly, the predicted MUN values from the model containing the weakness can be improved by applying an intercorrection factor for the effect of this primary component (i.e., protein) on MUN. The intercorrection factor would be applied as follows:

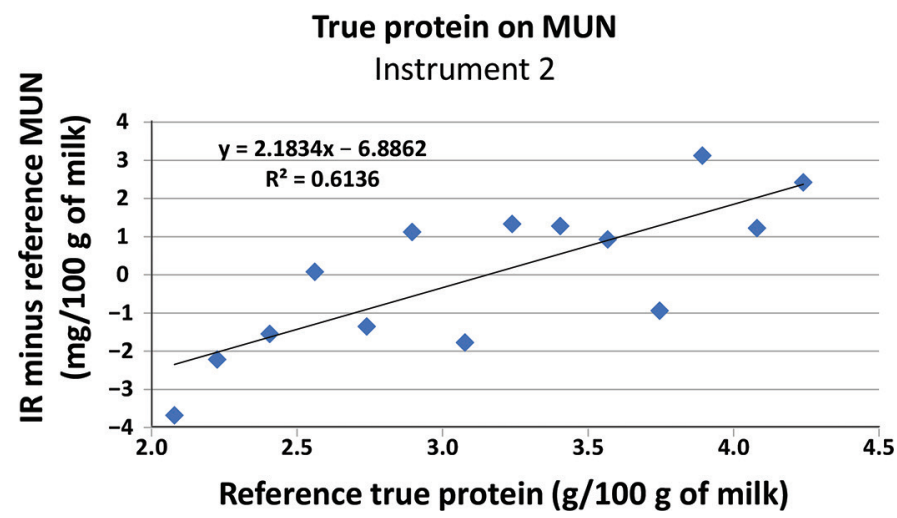

Figure 2. Partial least squares (PLS) model diagnostic graph for instrument 2, showing the difference between the MUN PLS model prediction value and the MUN reference value versus the reference chemistry concentration values for true protein of the modified milk calibration samples from April 29, 2019. IR = infrared.

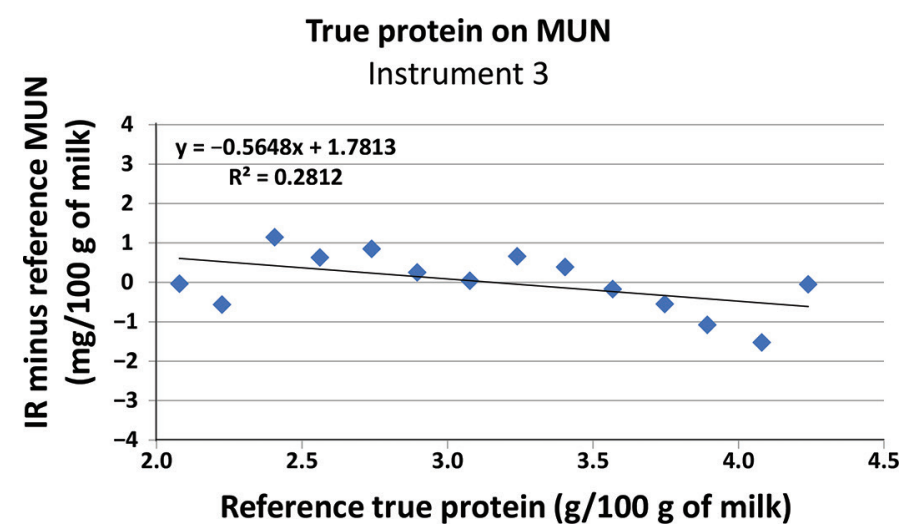

Figure 3. Partial least squares (PLS) model diagnostic graph for instrument 3, showing the difference between the MUN PLS model prediction value and the MUN reference value versus the reference chemistry concentration values for true protein of the modified milk calibration samples from April 29, 2019. IR = infrared.

$$
\mathrm{MUN}_{2}=\mathrm{MUN}_{1}+\mathrm{IC}_{\mathrm{PC}} \times \mathrm{PC}
$$

where $\mathrm{MUN}_{2}=$ intercorrected MUN value after applying the determined intercorrection factor; $\mathrm{MUN}_{1}=$ MUN value obtained from the PLS model containing a weakness; $\mathrm{IC}_{\mathrm{PC}}=$ intercorrection factor for the effect of the primary milk component on MUN; PC = concentration of the primary milk component. The calculated "intercorrected" $\mathrm{MUN}_{2}$ values would be secondary slope and intercept adjusted using the calibration sample set reference values just as in routine milk MIR calibration (Kaylegian et al., 2006) to obtain a final corrected MUN value as follows:

$$
\mathrm{MUN}_{3}=\mathrm{S} \times \mathrm{MUN}_{2}+\mathrm{B}
$$

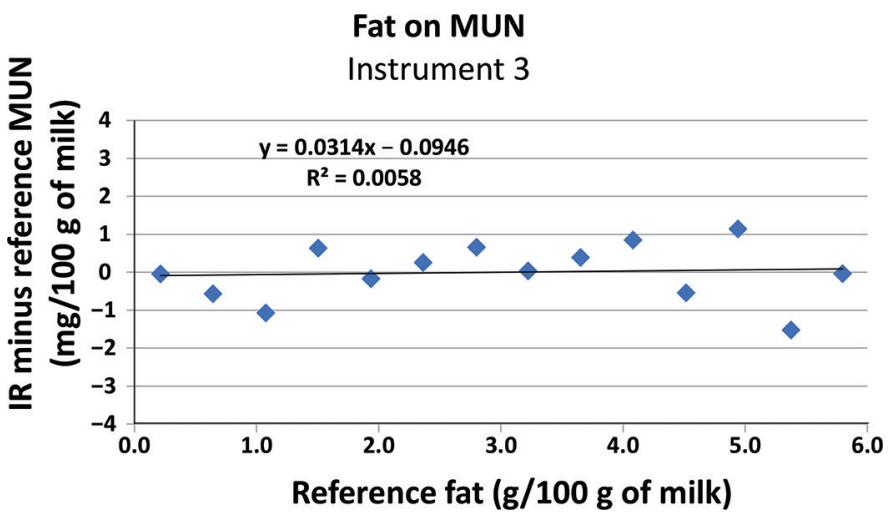

Figure 4. Partial least squares (PLS) model diagnostic graph for instrument 3, showing the difference between the MUN PLS model prediction value and the MUN reference value versus the reference chemistry concentration values for fat of the modified milk calibration samples from April 29, 2019. IR = infrared. 


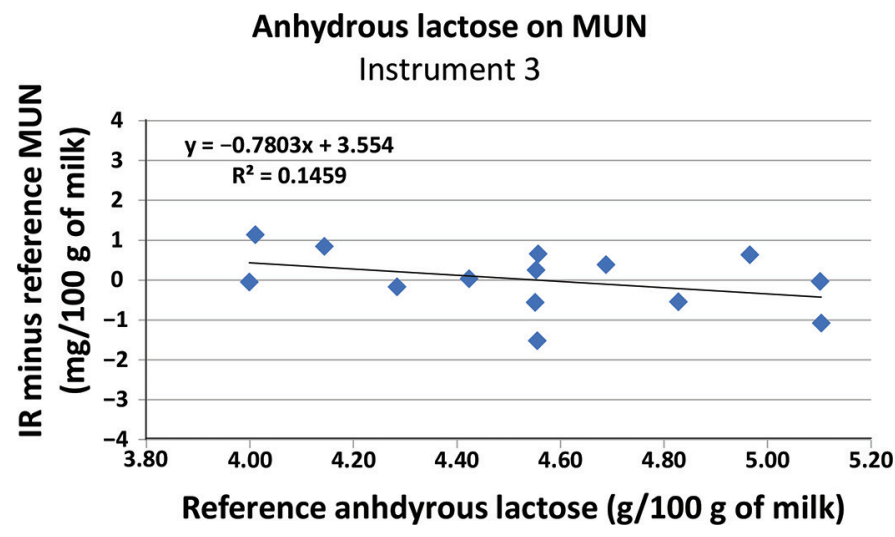

Figure 5. Partial least squares (PLS) model diagnostic graph for instrument 3, showing the difference between the MUN PLS model prediction value and the MUN reference value versus the reference chemistry concentration values for anhydrous lactose of the modified milk calibration samples from April 29, 2019. IR = infrared.

where $\mathrm{MUN}_{3}=$ corrected MUN value after applying slope and intercept adjustment; $\mathrm{MUN}_{2}=$ intercorrected MUN value; $\mathrm{S}=$ slope from the linear regression of the graph of $\mathrm{MUN}_{2}$ versus MUN reference values; B $=$ intercept from the linear regression of the graph of $\mathrm{MUN}_{2}$ versus MUN reference values. After application of the calculation for intercorrection described above to the data represented in Figure 2, the residual plot for MUN predictions by instrument 2 is shown in Figure 6 .

A demonstration of how a slope and intercept adjustment with the set of modified calibration samples improves the mean accuracy of a PLS model prediction by making the mean difference between the reference chemistry and instrument PLS model predictions (i.e., mean difference) equal zero is shown in Table 6 for all 3 instruments. The adjustment of slope and intercept of each instrument had very little effect on the standard deviation of the difference between instrument and reference chemistry for MUN because the standard deviation of the differences (SDD) is a function of the quality of the PLS model (i.e., a better PLS model will have a small SDD). The SDD between reference chemistry and instrument predictions for the 14 milks were
True protein on MUN Instrument 2

(after intercorrection and $\mathrm{S} / \mathrm{I}$ adjustment)

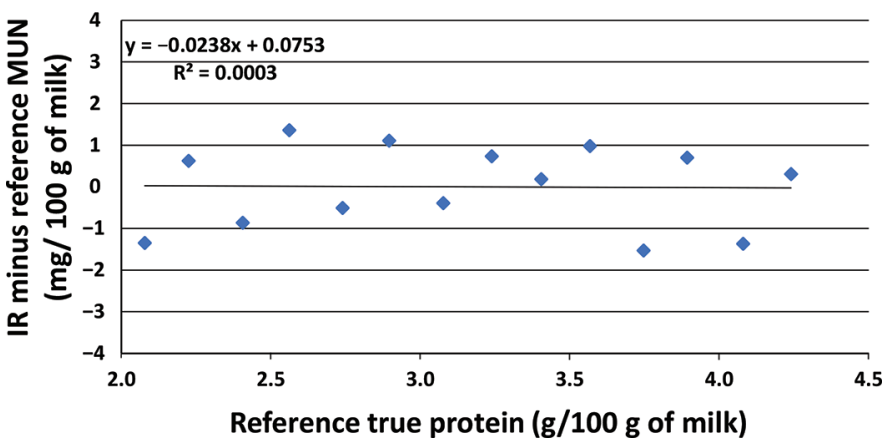

Figure 6. Partial least squares (PLS) model diagnostic graph for instrument 2, after true protein intercorrection factor and slope and intercept (S/I) adjustment were applied, showing the difference between the corrected MUN PLS model prediction value and the MUN reference value versus the reference chemistry concentration values for true protein of the modified milk calibration samples from April 29, 2019. IR $=$ infrared.

very different from one instrument to another (Table 6). Instrument 2 had an SDD that was more than twice that of the other 2 instruments and did not decrease after adjustment of the slope and intercept with the calibration samples (Table 6). The difference in SDD among the 3 instruments after calibration represented fundamental differences in the performance of the 3 different PLS models on these instruments. Using the data from Figure 2, an intercorrection factor was calculated for instrument 2 to correct for influence of background variation in true protein on the MUN prediction.

The intercorrection factor for the effect of background true protein variation on MUN and a slope and intercept adjustment were applied to the MUN predicted values from the PLS model from instrument 2 (which is shown in Figure 2). Figure 6 shows the PLS model diagnostic graph for the intercorrected and slope- and intercept-adjusted data from instrument 2. Applying the intercorrection factor to the data from instrument 2 and adjusting the slope and intercept improved the

Table 6. Mean (MD) and standard deviation (SDD) of the differences between the partial least squares model predicted MUN value and the chemical reference MUN value for each instrument before and after slope and intercept (S-I) adjustment, using the modified milk calibration sample set

\begin{tabular}{lccccc}
\hline & \multicolumn{2}{c}{ Before S-I adjustment } & & \multicolumn{2}{c}{ After S-I adjustment } \\
\cline { 2 - 3 } \cline { 5 - 6 } Instrument & $\mathrm{MD}$ & $\mathrm{SDD}$ & & $\mathrm{MD}$ & $\mathrm{SDD}$ \\
\hline 1 & -0.1921 & 0.6415 & & 0.0000 & 0.5934 \\
2 & -1.3224 & 1.9582 & & 0.0000 & 1.9481 \\
3 & 0.1029 & 0.7838 & & 0.0000 & 0.7444 \\
\hline
\end{tabular}


Table 7. Mean (MD) and standard deviation (SDD) of the difference between the partial least squares model predicted MUN value and the chemical reference MUN value for instrument 2 before and after a true protein intercorrection and slope and intercept (S-I) adjustment were applied to reduce SDD

\begin{tabular}{lccccc}
\hline & \multicolumn{2}{c}{ Before intercorrection and S-I adjustment } & & \multicolumn{2}{c}{ After protein intercorrection and S-I adjustment } \\
\cline { 2 - 3 } \cline { 5 - 6 } Item & MD & SDD & MD & 0.0004 & 0.9912 \\
\hline Instrument 2 & -1.3224 & 1.9582 & & SDD \\
\hline
\end{tabular}

performance of the instrument and reduced the SDD from 1.96 to $0.99 \mathrm{mg}$ of MUN/100 g of milk (Table 7).

\section{CONCLUSIONS}

The modified milk calibration sample set was redesigned to obtain a wide, well-distributed range of MUN by modification of the UF and formulation procedure to produce a 14-sample set where the variation in concentration of MUN was not correlated (i.e., orthogonal) with changes in the fat, protein, and lactose in the 14-milk sample set. The within- $\left(\mathrm{RSD}_{\mathrm{r}}\right)$ and between$\left(\mathrm{RSD}_{\mathrm{R}}\right)$ laboratory variation in the reference values for MUN were 0.43 and $0.77 \%$, respectively, and the average expanded analytical uncertainty for the mean MUN value of the 14-sample calibration set was 16.15 $\mathrm{mg} / 100 \mathrm{~g}$ of milk $\pm 0.09 \mathrm{mg} / 100 \mathrm{of} \mathrm{g}$ milk. After slope and intercept adjustment to achieve a mean difference of zero with the calibration samples, it could be seen that the SDD of predicted versus reference MUN values among 3 different instruments and their PLS models were quite different. The orthogonal sample set was used to determine when a PLS model did not correctly model out the background variation in fat, true protein, or anhydrous lactose, to calculate an intercorrection factor to eliminate that effect, and to improve the model performance (i.e., $50 \%$ reduction in SDD between instrument predictions and reference chemistry values for MUN).

\section{ACKNOWLEDGMENTS}

The authors thank all USDA Federal Milk Market laboratories (Test Procedures Committee, USDA Federal Milk Markets, Carrollton, TX) and affiliated laboratories for their collaboration and sample analysis in this work. The technical assistance in sample preparation by Michelle Bilotta, Sara Hatch, and Ashley Kosicky (Department of Food Science, Cornell University, Ithaca, NY) was important for the success of this project. The authors thank the Test Procedures Committee of the USDA, Dairy Programs, Federal Milk Markets for their financial support of this research. Use of names, names of ingredients, and identification of specific models of equipment is for scientific clarity and does not constitute any endorsement of product by authors, Cornell University, or the Northeast Dairy Foods Research Center. The authors have not stated any conflicts of interest.

\section{REFERENCES}

AOAC International. 2019. Official Methods of Analysis. 21st ed. AOAC International.

Barbano, D. M., J. L. Clark, and C. E. Dunham. 1988. Comparison of Babcock and ether extraction methods for determination of fat content of milk: Collaborative study. J. Assoc. Off. Anal. Chem. 71:898-914. https://doi.org/10.1093/jaoac/71.5.898.

Barbano, D. M., J. M. Lynch, and J. R. Fleming. 1991. Direct and indirect determination of true protein content of milk by Kjeldahl analysis: Collaborative study. J. Assoc. Off. Anal. Chem. 74:281288. https://doi.org/10.1093/jaoac/74.2.281.

Caplan, Z., C. Melilli, and D. M. Barbano. 2013. Gravity separation of fat, somatic cells, and bacteria in raw and pasteurized milks. J. Dairy Sci. 96:2011-2019. https://doi.org/10.3168/jds.2012-6006.

Ellison, S. L. R., M. Rosslein, and A. Williams. 2000. Pages 25-27 in Eurachem/CITAC Guide CG4: Quantifying Uncertainty in Analytical Measurement. Istituto Nazionale di Ricerca Metrologica (INRIM), Metrology for Quality of Life Division.

Ferguson, J. D., and W. Chalupa. 1989. Impact of protein nutrition on reproduction in dairy cows. J. Dairy Sci. 72:746-766. https://doi .org/10.3168/jds.S0022-0302(89)79168-2.

Geer, S. R., and D. M. Barbano. 2014. The effect of immunoglobulins and somatic cells on the gravity separation of fat, bacteria, and spores in pasteurized whole milk. J. Dairy Sci. 97:2027-2038. https://doi.org/10.3168/jds.2013-7629.

Goulden, J. D. S. 1964. Analysis of milk by infra-red absorption. J. Dairy Res. 31:273-284. https://doi.org/10.1017/S0022029900018203.

Haaland, D. M., and E. V. Thomas. 1988. Partial least-squares methods for spectral analyses. 1. Relation to other quantitative calibration methods and the extraction of qualitative information. Anal Chem. 60:1193-1202. https://doi.org/10.1021/ac00162a020.

Kaylegian, K. E., G. E. Houghton, J. M. Lynch, J. R. Fleming, and D. M. Barbano. 2006. Calibration of infrared milk analyzers: Modified milk versus producer milk. J. Dairy Sci. 89:2817-2832. https://doi .org/10.3168/jds.S0022-0302(06)72555-3.

Kaylegian, K. E., J. M. Lynch, J. R. Fleming, and D. M. Barbano. 2009. Influence of fatty acid chain length and unsaturation on midinfrared milk analysis. J. Dairy Sci. 92:2485-2501. https://doi.org/ 10.3168/jds.2008-1910.

Kohn, R. A., Z. Dou, J. D. Ferguson, and R. C. Boston. 1997. A sensitivity analysis of nitrogen losses from dairy farms. J. Environ. Manage. 50:417-428. https://doi.org/10.1006/jema.1997.0133.

Larson, S. F., W. R. Butler, and W. B. Currie. 1997. Reduced fertility associated with low progesterone post breeding and increased milk urea nitrogen in lactating cows. J. Dairy Sci. 80:1288-1295. https: //doi.org/10.3168/jds.S0022-0302(97)76058-2.

Lefier, D. 1996. International Dairy Federation Standard 315: UHT Cream - Analytical Methods for the Determination of Urea Con- 
tent in Milk - Transgenic Dairy Mammals - Oxidized Sterols. International Dairy Federation.

Lynch, J. M. 1998. Use of AOAC International method performance statistics in the laboratory. J. AOAC Int. 81:679-684. https://doi .org/10.1093/jaoac/81.3.679.

Lynch, J. M., D. M. Barbano, and J. R. Fleming. 2007. Determination of the lactose content of fluid milk by spectrophotometric enzymatic analysis using weight additions and path length adjustment: Collaborative study. J. AOAC Int. 90:196-216.

Lynch, J. M., D. M. Barbano, M. Schweisthal, and J. R. Fleming. 2006. Precalibration evaluation procedures for mid-infrared milk Analyzers. J. Dairy Sci. 89:2761-2774. https://doi.org/10.3168/jds .S0022-0302(06)72353-0.

Nousiainen, J., K. J. Shingfield, and P. Huhtanen. 2004. Evaluation of milk urea nitrogen as a diagnostic of protein feeding. J. Dairy Sci. 87:386-398. https://doi.org/10.3168/jds.S0022-0302(04)73178-1.

Rajala-Schultz, P. J., W. J. A. Saville, G. S. Frazer, and T. E. Wittum. 2001. Association between milk urea nitrogen and fertility in Ohio dairy cows. J. Dairy Sci. 84:482-489. https://doi.org/10.3168/jds .S0022-0302(01)74498-0.

Roseler, D. K., J. D. Ferguson, C. J. Sniffen, and J. Herrema. 1993. Dietary protein degradability effects on plasma and milk urea nitrogen and milk nonprotein nitrogen in Holstein cows. J. Dairy Sci. 76:525-534. https://doi.org/10.3168/jds.S0022-0302(93)77372-5.

Wojciechowski, K. L., C. Melilli, and D. M. Barbano. 2016. A proficiency test system to improve performance of milk analysis methods and produce reference values for component calibration samples for infrared milk analysis. J. Dairy Sci. 99:6808-6827. https:/ /doi.org/10.3168/jds.2016-10936.

\section{ORCIDS}

C. Coon (ํ) https://orcid.org/0000-0002-3576-3249

D. M. Barbano $\odot$ https://orcid.org/0000-0002-0206-7028 